Article

\title{
Tracking Control of Shape-Memory-Alloy Actuators Based on Self-Sensing Feedback and Inverse Hysteresis Compensation
}

\author{
Shu-Hung Liu, Tse-Shih Huang and Jia-Yush Yen* \\ Department of Mechanical Engineering, National Taiwan University, Taipei, 10617, Taiwan \\ E-Mails: b87502061@ntu.edu.tw (S.H.L.); tshuang@dlit.edu.tw (T.S.H.) \\ * Author to whom correspondence should be addressed; E-Mail: jyen@ntu.edu.tw; \\ Tel.: +886-2-33662688; Fax: +886-2-33662688.
}

Received: 18 November 2009; in revised form: 15 December 2009 / Accepted: 16 December 2009 /

Published: 28 December 2009

\begin{abstract}
Shape memory alloys (SMAs) offer a high power-to-weight ratio, large recovery strain, and low driving voltages, and have thus attracted considerable research attention. The difficulty of controlling SMA actuators arises from their highly nonlinear hysteresis and temperature dependence. This paper describes a combination of self-sensing and model-based control, where the model includes both the major and minor hysteresis loops as well as the thermodynamics effects. The self-sensing algorithm uses only the power width modulation (PWM) signal and requires no heavy equipment. The method can achieve high-accuracy servo control and is especially suitable for miniaturized applications.
\end{abstract}

Keywords: shape memory alloys; self-sensing control; hysteresis model

\section{Introduction}

Shape memory alloys (SMAs) are metals that can recover from strains of up to $10 \%$ via stress- or temperature-induced crystalline transformation between high-temperature austenite and low-temperature martensite phases [1]. The SMA actuator is constructed from a fiber-like SMA wire designed to contract and extend like real muscles. At room temperature, an SMA wire is soft and pliable, very much like a nylon thread. However, when heated it begins to contract sharply with a large force and eventually becomes as stiff as a piano wire. The maximum strain is typically $4.5 \%$ of its original length. When the SMA is again cooled to room temperature it softens and recovers its original length. 
Due to the characteristics of a high power-to-weight ratio, large recovery strain, and low driving voltages, the SMA actuator has been used in wide variety of applications including aircraft wing controls [2,3], robotic grippers [4-7], automotive mirror actuators [8], active vibration suppression [9], active endoscopes [10], and legged robots [11,12]. Most SMA applications require some form of length control, and a simple implementation involves using separate strain sensors for the SMA deformation for feedback control; however, this can be very difficult for some miniature applications and the sensorless approach offers an attractive alternative. The sensorless SMA control appraoches can be divided into two major categories. The first approach is to use the so-called self-sensing properties of the SMA actuator, whereby the change in the SMA electric resistance is measured to estimate the corresponding strain. Curve-fitting and a neural network have been used to model the SMA self-sensing properties [5,13]. These models were able to describe the major hysteresis loop of the SMA actuator but not the minor hysteresis loops. Most of the control applications also employed conventional PD control for the feedback action. The second approach to the sensorless SMA control uses no measurement feedback, but depends instead on mathematical models to estimate the SMA strain [14-16]; obviously this method is sensitive to the accuracy of the mathematical models.

In this paper, we propose a modified approach for precision sensorless SMA servo control that consists of three components: (1) a hysteresis model that combines the strengths of the two sensorless control strategies, (2) a thermodynamics model to compensate for the temperature effect, and (3) a spring model to include the strain energy effect. The hysteresis model is based on the Duhem differential model, and is used to describe both the major and minor hysteresis loops. A detailed model is necessary to fulfill the stringent precision control requirements. Variable supply voltages have previously been used to induce the SMA self-sensing relationship [5,13]. However, the resulting device (i.e., the programmable power supply) is far too large for miniature robot applications; this difficulty is overcome in this paper by using the PWM signal to obtain the self-sensing characteristics. This requires only a constant supply voltage, and so a bulky programmable power supply can be replaced by very small IC chips that are more suited for miniature applications. The PWM signal is also employed for controlling the SMA actuator displacements.

The Section 2 of the paper describes the experimental setup for the proposed control scheme. Section 3 explains the self-sensing property of the SMA actuator. Modeling of the SMA actuator is described in Section 4. The proposed scheme of tracking control based on self-sensing feedback and inverse hysteresis compensator is described in Section 5. Section 6 shows the experimental results. Finally, the conclusions are given in Section 7.

\section{Experimental Setup}

The main components of the experimental setup (a test platform and an electric circuit) are shown in Figure 1. The test platform was used to investigate the characteristics of a bias-type SMA wire actuator (the SMA wire contracts when heated, and it expands with the aid of the bias spring when cooled). A 167-mm-long NiTi-based SMA actuator with a diameter of $150 \mu \mathrm{m}$ (BMF150, TOKI) was installed on the test platform. One of the ends of the SMA actuator was fixed to the platform while the other end was connected to a moving slider. A pair of linear guides restricted the slider to move only in one 
dimension horizontally. The linear bias spring provided a restoring force to the SMA actuator. In addition, an LED displacement sensor (OMRON) was integrated to measure the displacement of the SMA actuator. Note that the displacement sensor was used in this study simply to validate the control results, and not to provide a feedback signal to the controller.

Figure 1. Experimental setup.

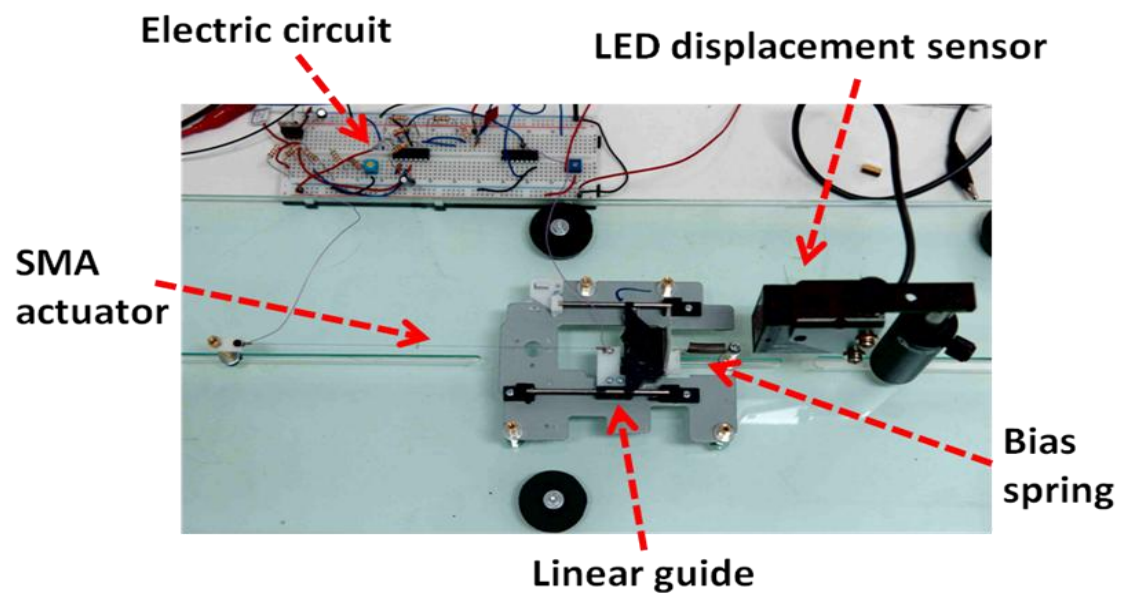

A schematic of the electric circuit is shown in Figure 2. A multifunction data acquisition card ( \pm 5 V full-scale range, 12-bit resolution; PCI-1711, Advantech) was employed to send the PWM signal via the digital output and measure the amplified voltage $V_{\text {amp }}$ via the analog input. A Darlington driver IC (ULN2003AP) was used as a switching element to control the heating or cooling state of the SMA actuator. A DC voltage source $V_{s}$ was connected to the SMA actuator to supply a DC voltage to heat the SMA actuator. A resistor, $R$, was connected serially to the SMA actuator to prevent it from overheating. VCE of the Darlington driver was then amplified by a differential amplifier to enlarge its variation due to the electric-resistance variation of the SMA actuator during the phase transformation process. The amplified voltage Vamp was measured by the data acquisition card.

Figure 2. Schematic of the electric circuit.

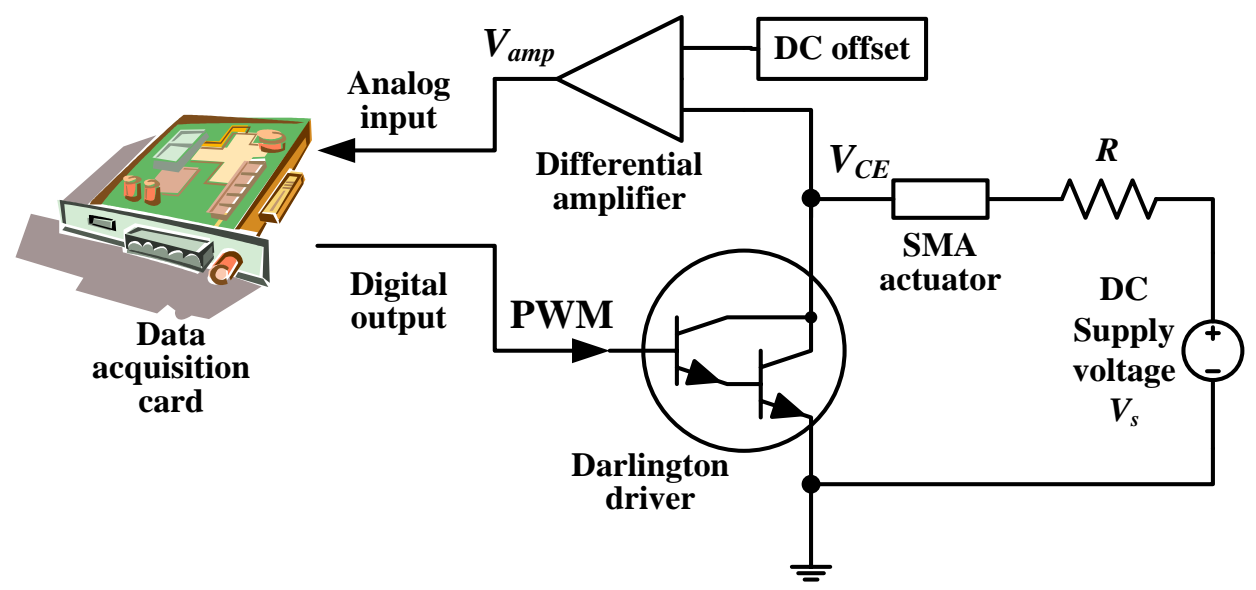




\section{Self-Sensing Property of SMA Actuator}

Figure 3 shows the relationship between the PWM signal that inputs to the Darlington driver and the measured $V_{\text {amp }}$. The supply voltage $V_{s}$ is set to $6 \mathrm{~V}$ and $100 \mathrm{~Hz}$ PWM signal with $40 \%$ duty ratio is input to the Darlington driver. It can clearly be seen in Figure 3 that during the "turn-off" state (low voltage level) of the input PWM signal, the corresponding $V_{a m p}$ is saturated at $5 \mathrm{~V}$. On the other hand, during the "turn-on" state (high voltage level) of the input PWM signal, the corresponding $V_{\text {amp }}$ drops to some steady value (in this case about $0.7 \mathrm{~V}$ ) after $1 \mathrm{~ms}$. At each falling edge of the input PWM signal, a $V_{\text {amp }}$ value is recorded as $V_{A}$. In other words, one $V_{A}$ is recorded during each PWM cycle. Note that it takes at least $1 \mathrm{~ms}$ for the $V_{\text {amp }}$ to drop from $5 \mathrm{~V}$ to a steady value; therefore, the duty ratio of the input $100 \mathrm{~Hz}$ PWM signal must higher than $10 \%$ in order to measurement the correct $V_{A}$.

Figure 3 shows the relationship between the PWM signal supplied to the Darlington driver and the measured $V_{\text {amp. }}$. The supply voltage $V_{s}$ was set to $6 \mathrm{~V}$ and a $100 \mathrm{~Hz}$ PWM signal with a $40 \%$ duty cycle was supplied to the Darlington driver. The figure clearly shows that $V_{a m p}$ saturated at $5 \mathrm{~V}$ during the "turn-off" state (low voltage level) of the input PWM signal, and dropped to some steady value (in this case about $0.7 \mathrm{~V}$ ) after $1 \mathrm{~ms}$ during the "turn-on" state (high voltage level). The $V_{\text {amp }}$ value at each falling edge of the input PWM signal is recorded as $V_{A}$; that is, one $V_{A}$ value is recorded during each PWM cycle. Note that it took at least $1 \mathrm{~ms}$ for the Vamp to drop from $5 \mathrm{~V}$ to a steady value; therefore, the duty cycle of the input $100-\mathrm{Hz}$ PWM signal had to be higher than $10 \%$ to allow the correct $V_{A}$ to be measured.

Figure 3. Relationship between $V_{a m p}$ (blue solid line), input PWM (red dashed line) and $V_{A}$ (circle).

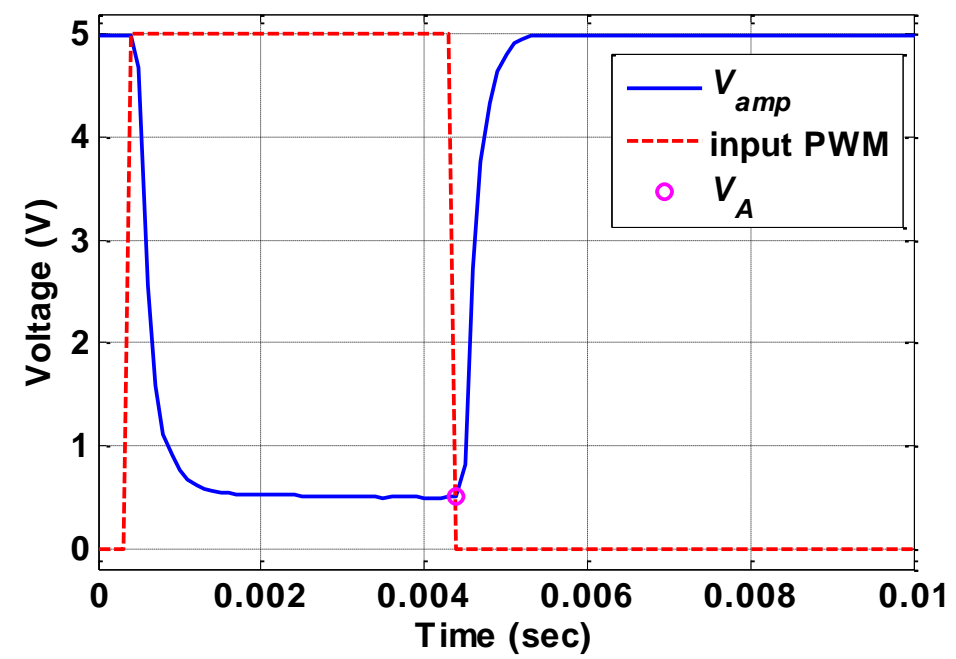

An open-loop experiment was performed to investigate the relationship between the contraction displacement of the SMA actuator $\left(D_{s}\right)$ and $V_{A}$. The room temperature was $23{ }^{\circ} \mathrm{C}$ and no additional cooling method was applied to the test platform. At the beginning of the experiment, the $100 \mathrm{~Hz}$ PWM signal with a $40 \%$ duty cycle was used as the input signal to slowly heat the SMA actuator. After the SMA actuator was fully contracted, the duty cycle of the input PWM signal was changed to $15 \%$. The SMA actuator was then cooled gradually. The values of $D_{s}$ and $V_{A}$ were recorded throughout the heating and cooling processes. The experiment was repeated 10 times, and Figure 4 shows the resulting 
plot of $D_{s}$ versus $V_{A}$. The data show high repeatability and are modeled in Section VI so as to characterize the self-sensing relationship of the SMA actuator. Note that the $D_{s}$-versus- $V_{A}$ plot varies with the pretension of the bias spring [13]. In this study, the pretension was set to $65 \mathrm{gw}$, and the spring constant $\left(k_{s}\right)$ of the bias spring was $35.035 \mathrm{~N} / \mathrm{m}$.

Figure 4. Plot of $D_{s}$ versus $V_{A}$ for 10 experiments.

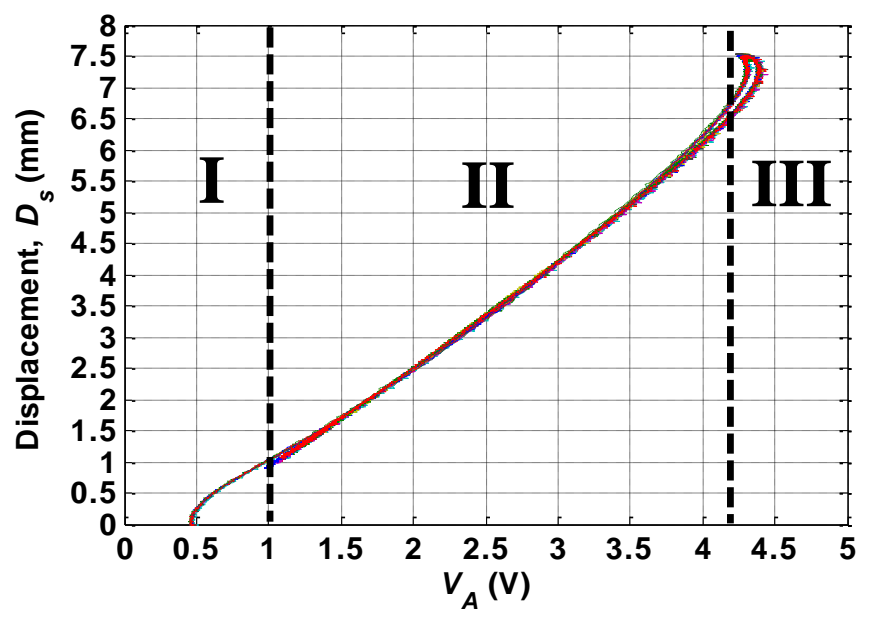

\section{Modeling of SMA Actuator}

\subsection{Modeling of Hysteresis}

An SMA actuator exhibits the hysteresis phenomenon [1]. Figrue 5 shows the relationship between temperature and strain of the BMF150 device as published in the product specifications [17]. For the heating/contraction process, the strain was small until the temperature was above $75{ }^{\circ} \mathrm{C}$, whereas for the cooling/elongation process a large strain appeared after the temperature decreased to below $70{ }^{\circ} \mathrm{C}$.

Figure 5. Relationship between strain and temperature for BMF150 [17].

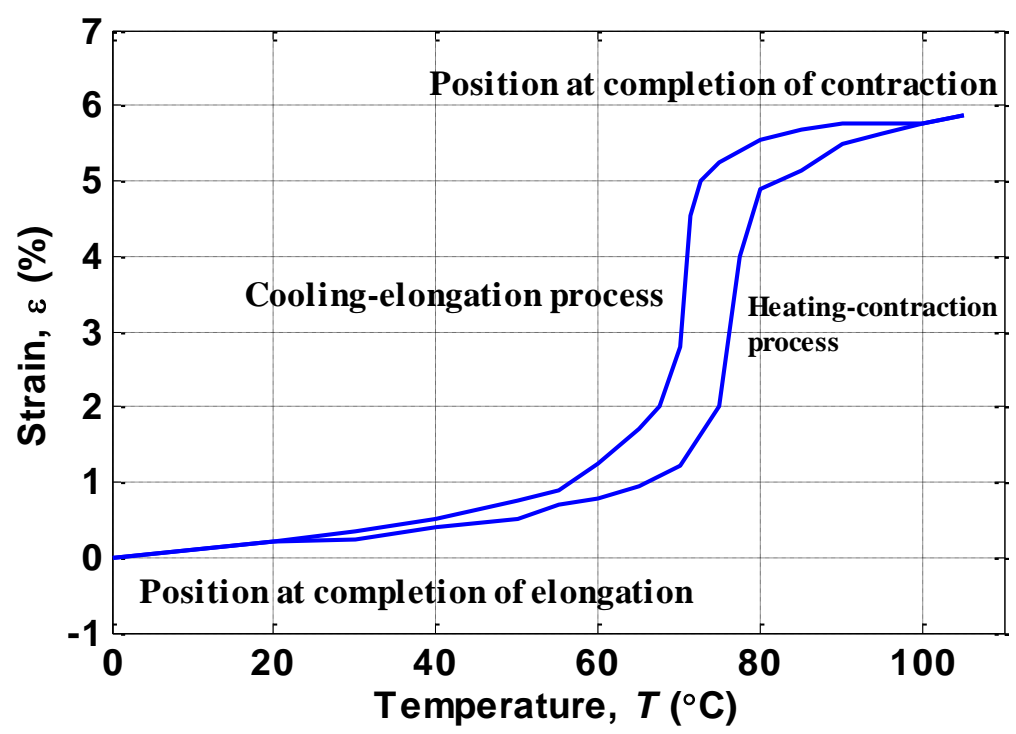


The Duhem differential model $[16,18]$ is used to model the hysteresis phenomenon of the SMA actuator:

$$
\left\{\begin{array}{c}
\dot{y}(t)=g_{+}(u(t), y(t))(\dot{u}(t))^{+}-g_{-}(u(t), y(t))(\dot{u}(t))^{-} \\
y(0)=y_{0},
\end{array}\right.
$$

where $u(t)$ denotes the input, $y(t)$ is the output, $y_{0}$ is the initial value of the output, $g_{ \pm}$is slope function, the subscripts + and - represent increasing and decreasing curves, respectively, and:

$$
(\dot{u}(t))^{ \pm}=(|\dot{u}(t)| \pm \dot{u}(t)) / 2
$$

Equation (2) indicates that $(\dot{u}(t))^{+}=\dot{u}(t)$ and $(\dot{u}(t))^{-}=0$ if $\dot{u}(t)>0$, and $(\dot{u}(t))^{+}=0$ and $(\dot{u}(t))^{-}=\dot{u}(t)$ if $\dot{u}(t)>0$. As a result, the slope function of (1) is $g_{+}$when $\dot{u}(t)>0$ and $g_{\text {- when }} \dot{u}(t)<0$.

The Gaussian combination membership function $\left(G_{C M F}\right)$ is chosen as the slope function of the hysteresis model. The $G_{C M F}$ is a combination of two Gaussian membership functions:

$$
G_{C M F}(u)=k_{C M F} \cdot G_{M F, 1}(u) \cdot G_{M F, 2}(u)
$$

where $k_{C M F}$ is the gain, $G_{M F, 1}(u)$ and $G_{M F, 2}(u)$ are the modified Gaussian membership functions, and is defined as:

$$
\begin{gathered}
G_{M F, 1}(u)=\left\{\begin{array}{cc}
\left(1-c_{1}\right) \exp \left(-\frac{\left(u-\mu_{1}\right)^{2}}{2 \sigma_{1}^{2}}\right)+c_{1}, & \text { if } u \leq \mu_{1} \\
1, & \text { if } u>\mu_{1}
\end{array}\right. \\
G_{M F, 2}(u)=\left\{\begin{array}{cc}
1, & \text { if } u \leq \mu_{2} \\
\left(1-c_{2}\right) \exp \left(-\frac{\left(u-\mu_{2}\right)^{2}}{2 \sigma_{2}^{2}}\right)+c_{2}, & \text { if } u>\mu_{2}
\end{array}\right.
\end{gathered}
$$

where $\mu_{i}$ denotes the mean, $\sigma_{i}^{2}$ denotes the variance, and $c_{i}$ is an offset value. Figure 6 gives an example of a $G_{C M F}$ based on two Gaussian membership functions: $G_{M F, 1}$ with $\mu_{1}=20, \sigma_{1}^{2}=9, c_{1}=0.1$, and $G_{M F, 2}$ with $\mu_{2}=60, \sigma_{2}^{2}=100, c_{2}=0.1$. The hysteresis between strain $\varepsilon$ and temperature $T$ shown in Figure 5 is modeled by the differential equation:

$$
\frac{d \varepsilon}{d T}= \begin{cases}G_{C M F,+}(T), & \text { if } \dot{T} \geq 0 \\ G_{C M F,-}(T), & \text { if } \dot{T}<0\end{cases}
$$

where $G_{C M F,+}$ and $G_{C M F,-}$ are the slope functions for heating and cooling curves, respectively. Figure 7 compares the hysteresis phenomenon of the SMA actuator between the device specifications and the model given by (6). The corresponding modeling parameters are listed in Table 1. 
Figure 6. Example of a Gaussian combination membership function (dotted line) based on two modified Gaussian membership functions $G_{M F, 1}$ (solid line) and $G_{M F, 2}$ (dashed line).

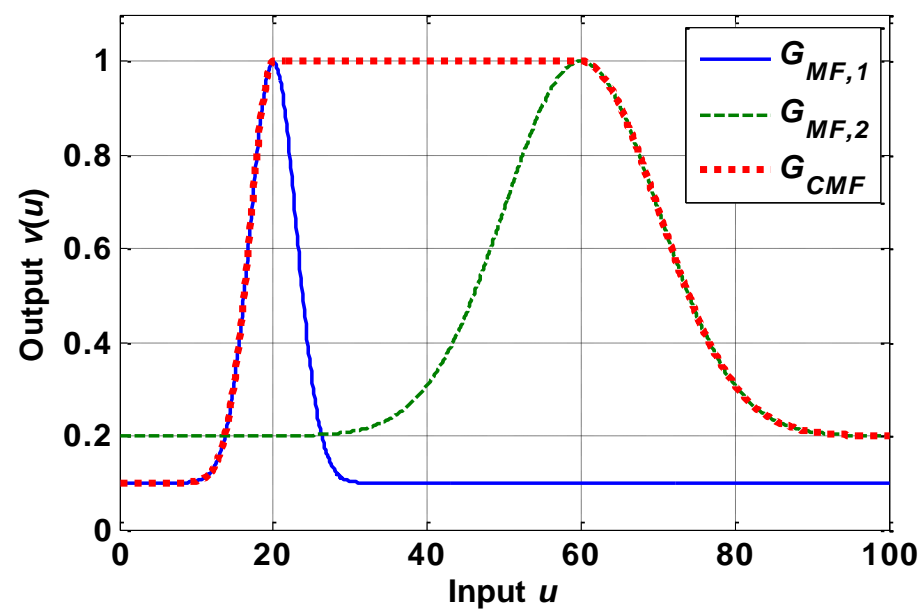

Figure 7. Comparison of the hysteresis of the SMA actuator between the device specifications (solid line) and the modeling result (dashed line).

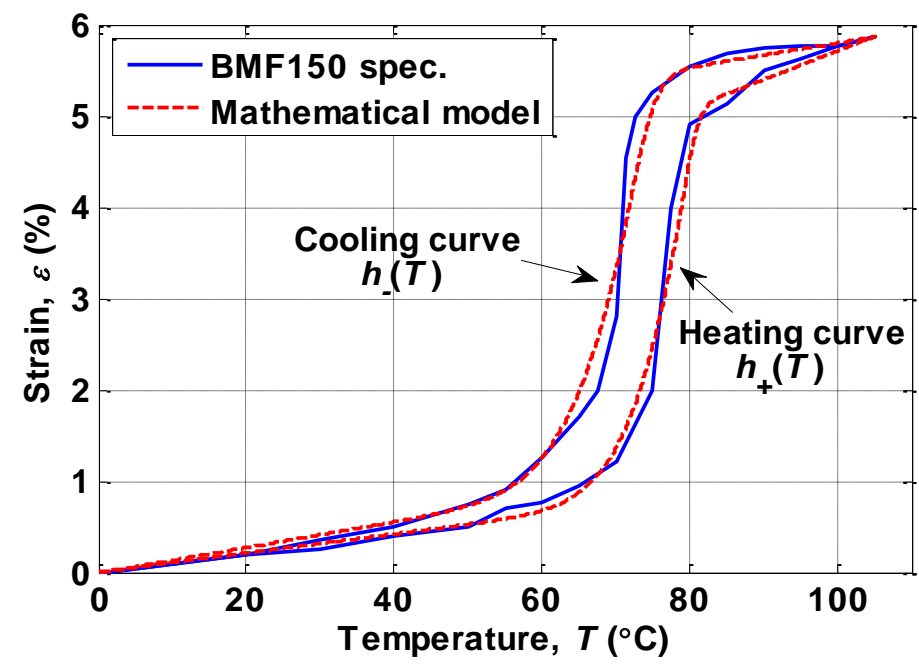

Table 1. Parameters for SMA hysteresis modeling.

\begin{tabular}{cccc}
\hline$\sigma_{1,+}^{2}$ & $10{ }^{\circ} \mathrm{C}^{2}$ & $\sigma_{1,-}^{2}$ & $12{ }^{\circ} \mathrm{C}^{2}$ \\
$\mu_{1,+}$ & $88{ }^{\circ} \mathrm{C}$ & $\mu_{1,-}$ & $84.6{ }^{\circ} \mathrm{C}$ \\
$c_{1,+}$ & $0.015 \%$ & $c_{1,-}$ & $0.02 \%$ \\
\hline$\sigma_{2,+}^{2}$ & $1.8{ }^{\circ} \mathrm{C}^{2}$ & $\sigma_{2,-}^{2}$ & $3{ }^{\circ} \mathrm{C}^{2}$ \\
$\mu_{2,+}$ & $78.65{ }^{\circ} \mathrm{C}$ & $\mu_{2,-}$ & $71{ }^{\circ} \mathrm{C}$ \\
$c_{2,+}$ & $0.045 \%$ & $c_{2,-}$ & $0.019 \%$ \\
\hline$k_{C M F,+}$ & 0.7 & $k_{C M F,-}$ & 0.7 \\
\hline
\end{tabular}


To model the minor hysteresis loops, (6) is modified by multiplying the slope function by a gain:

$$
\frac{d \varepsilon}{d T}= \begin{cases}\frac{h_{-}(T)-\varepsilon}{h_{-}(T)-h_{+}(T)} G_{C M F,+}(T), & \text { if } \dot{T} \geq 0 \\ \frac{\varepsilon-h_{+}(T)}{h_{-}(T)-h_{+}(T)} G_{C M F,-}(T), & \text { if } \dot{T}<0\end{cases}
$$

where $h_{+}(T)$ and $h_{-}(T)$ are the increasing and decreasing curves of the major hysteresis loop computed by (6), respectively. The simulated hysteresis model of the SMA actuator based on (7) is shown in Figure 8.

Figure 8. Simulated hysteresis characteristics of SMA actuator.

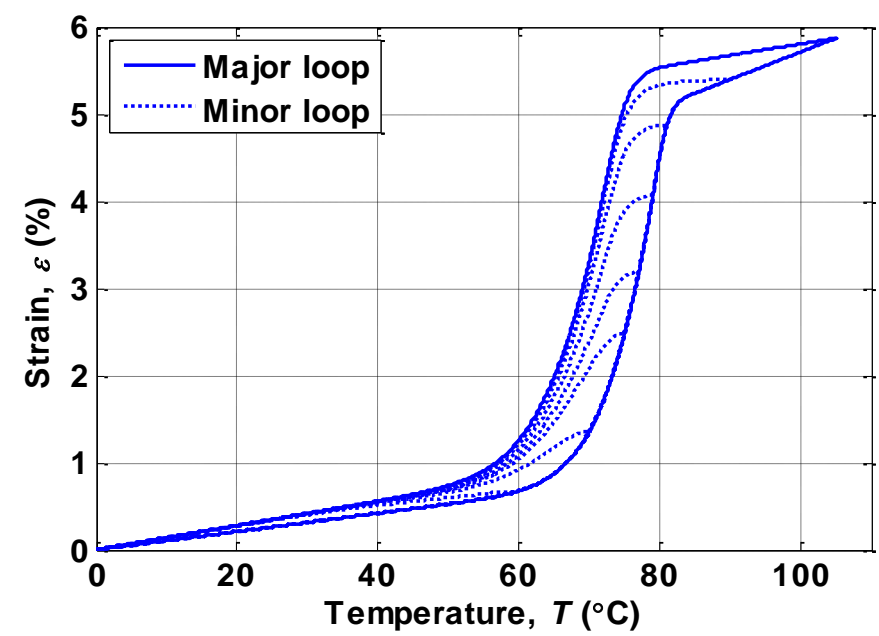

\subsection{Modeling of Inverse Hysteresis}

An inverse hysteresis model has to be derived to compensate for the hysteresis of the SMA actuator. We derived the following inverse hysteresis model by inverting the hysteresis differential equation (Equation 7) [19]:

$$
\frac{d T}{d \varepsilon}= \begin{cases}\frac{h_{-}(T)-h_{+}(T)}{h_{-}(T)-\varepsilon+\delta} \frac{1}{G_{C M F,+}(T)+\delta}, & \text { if } \dot{\varepsilon} \geq 0 \\ \frac{h_{-}(T)-h_{+}(T)}{\varepsilon-h_{+}(T)+\delta} \frac{1}{G_{C M F,-}(T)+\delta}, & \text { if } \dot{\varepsilon}<0\end{cases}
$$

where $\delta$ is a positive arbitrarily small constant that allows the differential equation to be solved. Figure 9 illustrates the simulated inverse hysteresis model of the SMA actuator based on (8). 
Figure 9. Simulated inverse hysteresis characteristics of SMA actuator.

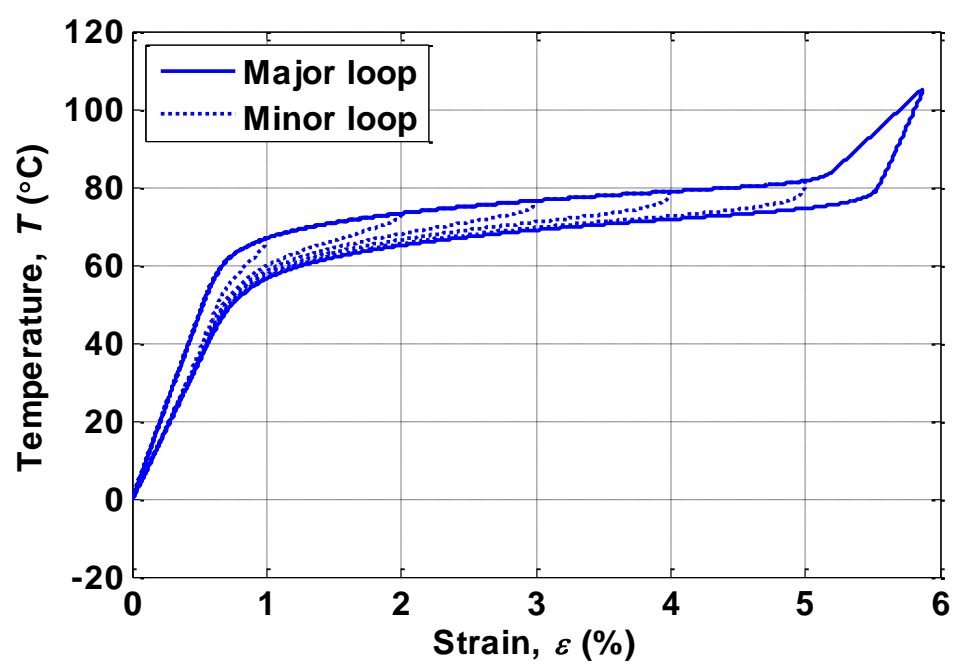

\subsection{Modeling of Temperature Dynamics}

The temperature dynamics are modeled by the heat transfer equation, which balances the heat across the SMA actuator. According to the Joule effect, the SMA actuator is heated when electric current is passed through it. We assume that heat loss occurs only via natural convection. The temperature dynamics are given by the following differential equation $[14,16,20]$ :

$$
\dot{T}=\frac{1}{m c}\left(P_{T}-\pi d_{s} L_{0} h\left(T-T_{a m b}\right)\right)
$$

where $T_{a m b}$ is the ambient temperature; $T, m, c, d_{s}$, and $L_{0}$ are the temperature, mass, specific heat, diameter, and undeformed length of the SMA actuator, respectively; $P_{T}$ is the electric power required to change the temperature of the SMA actuator; and $h$ is the coefficient of convectional heat transfer. Note that $h$ for a thin metal wire under natural cooling varies with temperature [21], and so its value was obtained by the method provided in [21] under the following conditions: $T_{a m b}=23{ }^{\circ} \mathrm{C}$, $d_{s}=1.5 \times 10^{-4} \mathrm{~m}, L_{0}=0.167 \mathrm{~m}$, and an air pressure of $1 \mathrm{~atm}$. Using the MATLAB curve-fitting tool, the relationship between heat convection coefficient $h$ and temperature $T$ is represented as:

$$
h(T)=a_{1} e^{a_{2} T}+b_{1} e^{b_{2} T}
$$

where $a_{1}=85.28, a_{2}=0.001727, b_{1}=-106.4$, and $b_{2}=-0.08706$. In addition, the specific heat of the SMA actuator does not remain constant during the actuation process, instead differing between the martensite phase $\left(c_{m}\right)$ and the austenite phase $\left(c_{a}\right)$; accordingly, the value of $c$ is based on the percentage of martensite transformation:

$$
c=\frac{L}{L_{0}} c_{m}+\left(1-\frac{L}{L_{0}}\right) c_{a}
$$

where $L_{0}$ is the undeformed length of the SMA actuator and $L$ is the actual length of the SMA actuator $\left(L=L_{0}-D_{s}\right)$. 


\subsection{Electric Power Calculation}

The electric power provided by the power source not only heats the bias-type SMA actuator but also supplies energy to elongate the bias spring. The total power, $P$, is given by:

$$
P=P_{T}+P_{W}
$$

where $P_{T}$ is the electric power required to change the temperature of the SMA actuator, as given in (9), and $P_{W}$ is the work required to provide the elastic potential energy to the bias spring, which is derived from the potential energy of the bias spring according to:

$$
\begin{aligned}
W & =\frac{1}{2} D_{s}\left[k_{s} l_{0}+k_{s}\left(l_{0}+D_{s}\right)\right] \\
& =k_{s} l_{0} D_{s}+\frac{1}{2} k_{s} D_{s}^{2}
\end{aligned}
$$

where $k_{s}$ is the spring constant of the bias-spring, $D_{s}$ is the displacement of the SMA actuator, and $l_{0}$ is the initial length of the bias-spring. $P_{W}$ is given by:

$$
P_{W}=\dot{W}=k_{s}\left(D_{s}+l_{0}\right) \dot{D}_{s}
$$

The duty cycle of the PWM signal $D$ that is supplied to the SMA actuator can then be estimate as

$$
D=\sqrt{\frac{P}{V_{P W M} I_{P W M}}}
$$

where $V_{P W M}$ is the high-level voltage of the PWM signal and $I_{P W M}$ is the electric current across the SMA actuator when $D=100 \%$.

\subsection{Modeling of Self-Sensing Properties}

To model the self-sensing properties of the SMA actuator, the curve of $D_{s}$ versus $V_{A}$ shown in Figure 4 is separated into three phases. In phases I and II, the mathematical models are obtained using the curve-fitting technique. The two heating curves in phases I and II, and the cooling curve in phase II are modeled respectively by the following equations:

$$
\begin{gathered}
D_{s}=f\left(V_{A}\right)=-0.8554 V_{A}^{2}+2.7850 V_{A}-0.9077 \\
D_{s}=f\left(V_{A}\right)=0.0192 V_{A}^{3}+0.0081 V_{A}^{2}+1.2960 V_{A}-0.3065 \\
D_{s}=f\left(V_{A}\right)=0.0811 V_{A}^{2}+1.3160 V_{A}-0.4681
\end{gathered}
$$

The $D_{S}$-versus- $V_{A}$ curve in Figure 4 exhibits slight hysteresis. However, the mathematical models given in (15)-(17) only describe the major hysteresis loop. The Duhem differential model is therefore employed to obtain the complete model including both major and minor hysteresis loops. The self-sensing properties of the SMA actuator are given by: 


$$
\frac{d D_{s}}{d V_{A}}= \begin{cases}\frac{h_{-}\left(V_{A}\right)-D_{s}}{h_{-}\left(V_{A}\right)-h_{+}\left(V_{A}\right)} S_{+}\left(V_{A}\right), & \text { if } \dot{V}_{A} \geq 0 \\ \frac{D_{s}-h_{+}\left(V_{A}\right)}{h_{-}\left(V_{A}\right)-h_{+}\left(V_{A}\right)} S_{-}\left(V_{A}\right), & \text { if } \dot{V}_{A}<0\end{cases}
$$

where $S_{+}\left(V_{A}\right)$ and $S_{-}\left(V_{A}\right)$ are the slope functions of the increasing and decreasing curves, respectively, which can be obtained by differentiating (15)-(17) once. $h_{+}\left(V_{A}\right)$ and $h_{-}\left(V_{A}\right)$ are the increasing and decreasing curves, respectively, of the major loop computed by the following equation:

$$
\frac{d D_{s}}{d V_{A}}= \begin{cases}S_{+}\left(V_{A}\right), & \text { if } \dot{V}_{A} \geq 0 \\ S_{-}\left(V_{A}\right), & \text { if } \dot{V}_{A}<0\end{cases}
$$

Figure 10 shows the mathematical model comparing with the experimental data.

Figure 10. Comparison of simulation and experimental results for the $D_{s}$-versus- $V_{A}$ curve.

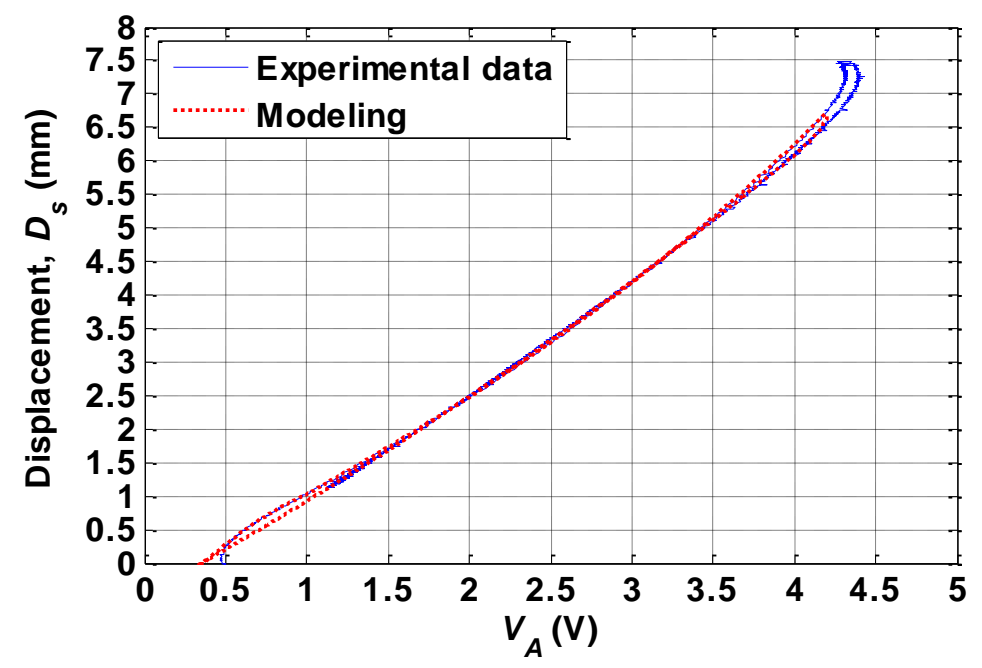

\section{Tracking Control with Self-Sensing Feedback and Inverse Compensation}

The SMA actuator is a highly nonlinear system owing to its hysteresis characteristics; accordingly, a feedforward inverse compensator is designed to compensate the hysteresis. A schematic of the inverse compensator for controlling the displacement of the SMA actuator is depicted in Figure 11. The input of the inverse compensator is the reference strain, $\varepsilon$, where $\varepsilon=D_{s} / L_{0}$. Block I outputs the corresponding temperature of the SMA actuator to block II, which computes the power $\left(P_{T}\right)$ required to heat (or cool) the SMA actuator to the specific temperature. Block III computes the power $\left(P_{W}\right)$ required to deform the bias spring. Total power $P\left(=P_{T}+P_{W}\right)$ is supplied to block IV to calculate the corresponding duty cycle $D$ of the input PWM signal. In summary, the feedforward inverse compensator estimates the duty cycle of the input PWM signal required to heat (or cool) the SMA actuator to the desired length. 
Figure 11. Schematic block diagram of the feed-forward inverse compensator for SMA length control.

Inverse compensator

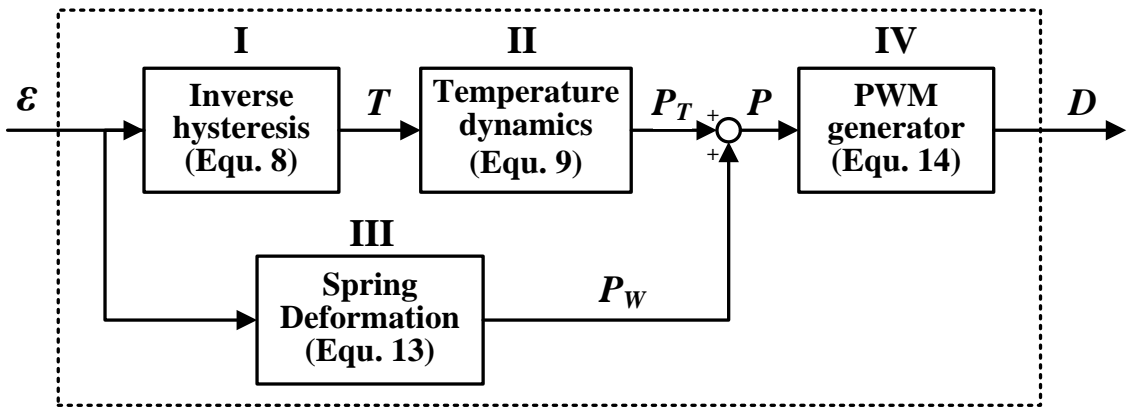

Figure 12 presents the control flow diagram of the proposed self-sensing feedback control with inverse hysteresis compensation. The strain estimated by the self-sensing characteristics described in (18) is used as the feedback source to compare with the reference strain. The error is supplied to a conventional PID controller to generate the appropriate duty cycle, $D_{2}$, of the PWM signal to implement the tracking ability. In addition, the reference strain is supplied to the feedforward inverse compensator to estimate the duty cycle, $D_{1}$, required for the reference strain. The SMA actuator is controlled by the PWM signal with duty cycle $D$ computed by adding $D_{1}$ and $D_{2}$.

Figure 12. Flow diagram of the SMA actuator length control.

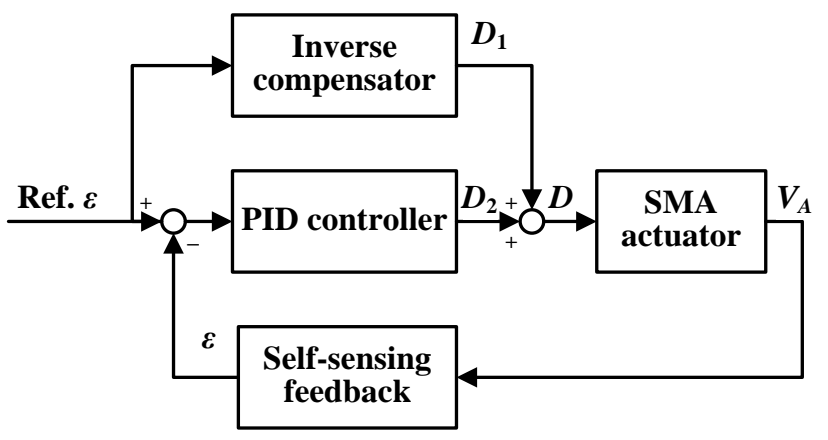

\section{Experimental Results}

Two experiments were performed to examine the tracking performance of the proposed control architecture. The first experiment used a sinusoidal reference signal. Figure 13 presents the experimental measurements from the displacement sensor, showing the reference sinusoidal signal and the results obtained using only the PID controller and simultaneously using both the PID controller and the feedforward inverse hysteresis compensator. The parameters of the PID controller were identical in both cases. Figure 13 shows clearly that the tracking error was large for the PID controller only when the reference signal switches between heating and cooling. This was attributed to by the very different heating and cooling hysteresis curves. Such errors reduced when the feedforward inverse hysteresis compensator was incorporated with the PID controller since this compensates the hysteresis of the SMA actuator. The RMS values of the tracking errors are listed in Table 2. 
Figure 13. Experimental results for a sinusoidal reference input.

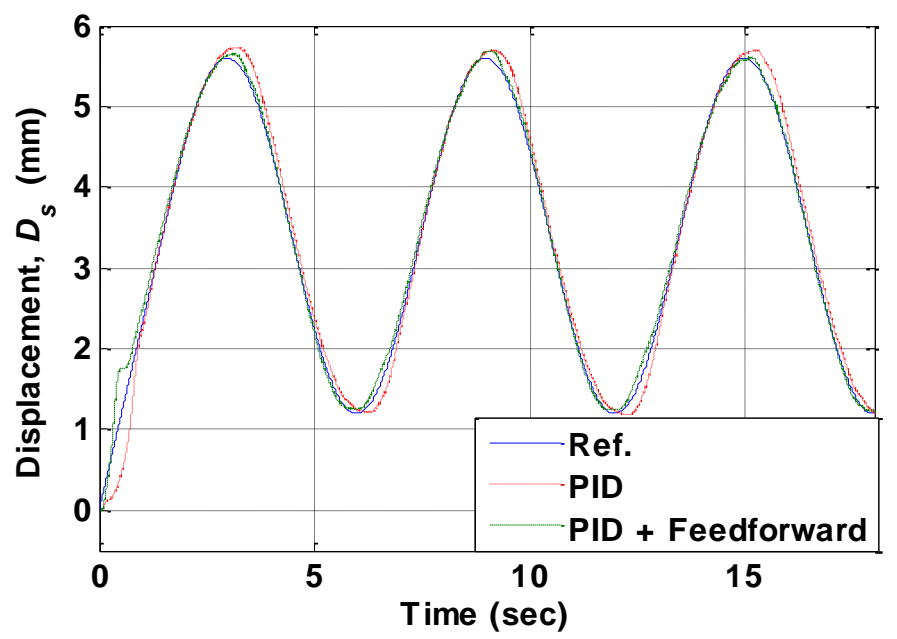

The second experiment used a multistep reference signal. The experimental results measured by the displacement sensor are shown in Figure 14, which indicates that when using only the PID controller the displacement trajectory could not follow the reference well at the beginning $(0 \sim 2 \mathrm{~mm})$ of the experiment. This was due to the nonlinearity of the SMA actuator shown in Figure 5 the temperature of the SMA actuator needed to increase about $65^{\circ} \mathrm{C}$ to move from 0 to $1 \%$ strain, but it only needed an increase of $35{ }^{\circ} \mathrm{C}$ for the SMA actuator to move from $1 \%$ to about $5.7 \%$ strain. However, the tracking errors improved when the feedforward inverse hysteresis compensator was incorporated with the PID controller. Note that the parameters of the PID controller were identical when using the PID controller alone or the combination of the PID controller and the feedforward inverse hysteresis compensator. The RMS values of the tracking errors are listed in Table 2.

Figure 14. Experimental results for a multi-step reference input.

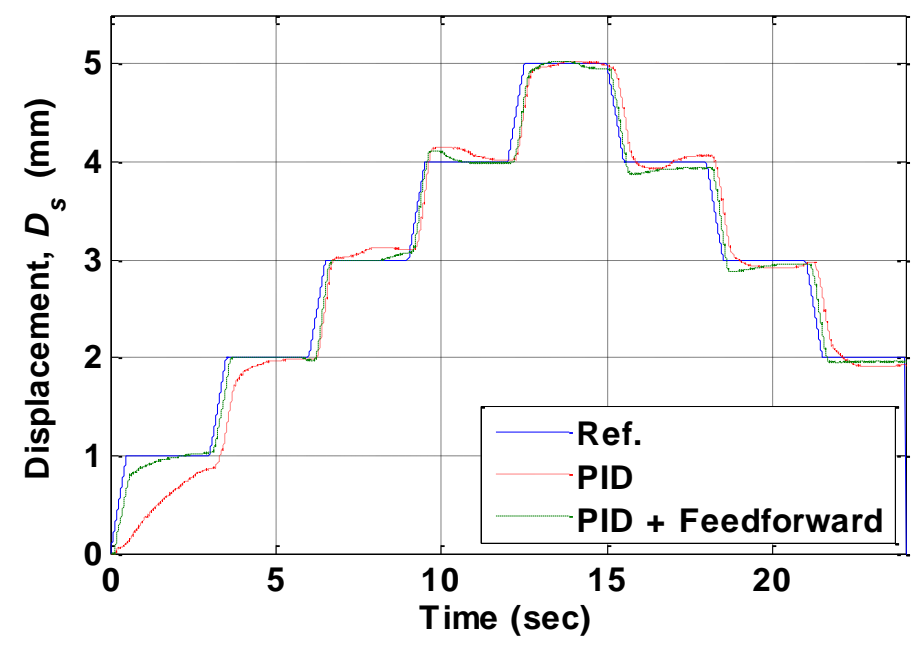

As a comparison, Figure 15 showed the same control with different models: the Duhem model, the major loop model proposed in [13], and the single curve model proposed in [22]. The major loop model merely modeled the major hysteresis loop and thus resulted in rapid chattering in the control effort when the actuator response approaches steady state. This was due to the fact that the underlying model 
switched between the "heating" and the "cooling" curves. To avoid this difficulty, the single loop model used only a single polynomial for the self-sensing characteristics. The Duhem differential model also suppressed the chattering behavior. The RMS tracking error for the major loop model, the single curve model, and the Duhem model were $0.2890,0.2781$, and $0.2311 \mathrm{~mm}$, as shown in Table 2. It could be seen that the control based on the Duhem model and teh single curve model exhibited larger transient responses than the control with the proposed model, which achieved an RMS error of $0.1223 \mathrm{~mm}$.

Figure 15. PID control with different self-sensing models: the single curve model (dash-dotted line), the major loop model (dashed line), and the Duhem model (solid line).

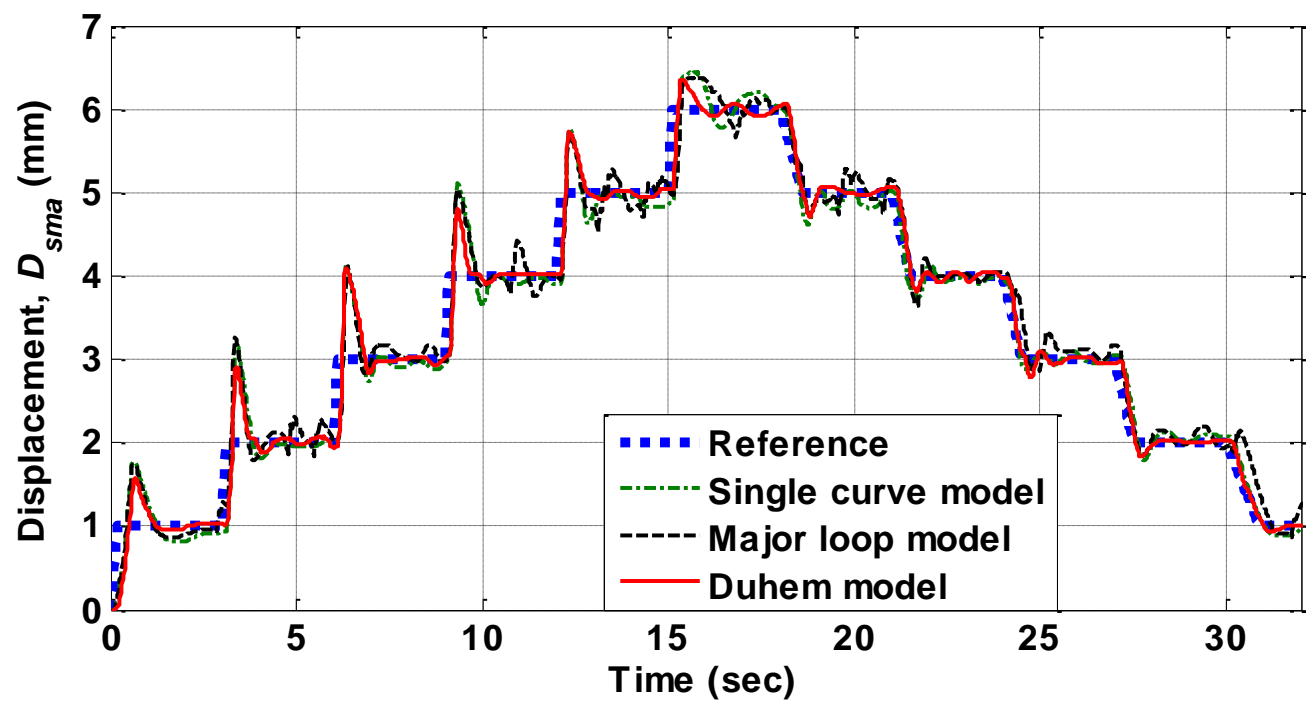

Table 2. RMS errors of the tracking errors.

RMSE Single curve model Major loop model Duhem model Proposed model

\begin{tabular}{lllll}
\hline$(\mathrm{mm})$ & 0.2781 & 0.2890 & 0.2311 & 0.1223 \\
\hline
\end{tabular}

\section{Conclusions and Future Remarks}

This paper described a PWM based self-sensing feedback controller with inverse hysteresis compensator for a SMA actuator. The proposed SMA compensator comprised an inverse hysteresis model to represent the major and minor hysteresis loops, a temperature dynamics model to compute the required input power to heat up the SMA actuator, and a spring force model that took accounted for the strain energy required to deform the actuator. The inverse hysteresis model was based on the Duhem self-sensing characteristic represented by the $D_{s}-V_{A}$ relationship. Both the major hysteresis loop and the minor hysteresis loops were considered. As a result, the model enabled accurate estimate of the actuator strain by using the electrical potential across the actuator. Experimental results showed that the self-sensing model achieved smaller transient error and can effectively suppress the chattering phenomenon.

It is worth noting that the current control scheme depends on the precise knowledge of the ambient temperature and the material properties. While the material properties remain mostly constants, the ambient temperature may experience unexpected changes. Future research will address the 
robustness issues of the proposed control against unknown ambient temperature changes and material property variations.

\section{Acknowledgement}

This paper is supported by the National Science Council, Taiwan under grant No. NSC94-2213-E002-021 and No. NSC98-2811-E-002-136.

\section{References}

1. Smith, R.C. Smart Material Systems; Society for Industrial and Applied Mathematics: Philadelphia, PA, USA, March 2005.

2. Kudva, J.N.; Sanders, B.P.; Pinkerton-Florance, J.L.; Garcia, E. Overview of the DARPA/ AFRL/NASA smart wing phase II program. In Smart Structures and Materials 2001: Industrial and Commercial Applications of Smart Structures Technologies; SPIE-International Society for Optical Engine: Newport Beach, CA, USA, 2001; pp. 383-389.

3. Song, G.B.; Ma, N. Robust control of a shape memory alloy wire actuated flap. Smart Mater. Struct. 2007, 16, N51-N57.

4. Yan, S.; Liu, X.; Xu, F.; Wang, J. A gripper actuated by a pair of differential SMA springs. J. Intel. Mat. Syst. Struct. 2007, 18, 459-466.

5. Zhang, H.; Bellouard, Y.; Burdet, E.; Clavel, R.; Poo, A.N.; Hutamacher, D.W. Shape memory alloy microgripper for robotic microassembly of tissue engineering scaffolds. In Proceedings of the 2004 IEEE International Conference on Robotics and Automation, New Orleans, LA, USA, April 2004, pp. 4918-4924.

6. Ashrafiuon, H.; Jala, V.R. Sliding mode control of mechanical systems actuated by shape memory alloy. J. Dyn. Syst., Meas. Contr. 2009, 131, 011010-011016.

7. Yang K.; Gu, C.L. A novel robot hand with embedded shape memory alloy actuators. Proc. IME C J. Mech. Eng. Sci. 2002, 216, 737-745.

8. Williams, E. Elahinia, M.H. An automotive SMA mirror actuator: modeling, design, and experimental evaluation. J. Intel. Mat. Syst. Struct. 2008, 19, 1425-1434.

9. Dhanalakshmi, K.; Umapathy, M. Active vibration control of SMA actuated structures using fast output sampling based sliding mode control. Instrum. Sci. Technol. 2008, 36, 180-193.

10. Ikuta, K.; Tsukamoto, M.; Hirose, S. Shape memory alloy servo actuator system with electric resistance feedback and application for active endoscope. In Proceedings of IEEE International Conference on Robotics and Automation, Philadelphia, PA, USA, April 1988; pp. 427-430.

11. Liu, S.H.; Yen, J.Y. A hexapod robot based on shape memory alloy actuators. In Proceedings of the 4th IFAC-Symposium on Mechatronic Systems, Heidelberg, Germany, September 2006; pp. 689-693.

12. Tu, K.Y.; Lee, T.T.; Wang, C.H.; Chang, C.A. Design of a fuzzy walking pattern (FWP) for a shape memory alloy (SMA) biped robot. Robotica 1999, 17, 373-382. 
13. Song, G.B.; Ma, N.; Lee, H.J.; Position estimation and control of SMA actuators based on electrical resistance measurement. Smart Struct. Syst. 2007, 3, 189-200.

14. Jayender, J.; Patel, R.V.; Nikumb, S.; Ostojic, M. Modeling and control of shape memory alloy actuators. IEEE Trans. Control Syst. Technol. 2008, 16, 279-287.

15. Majima, S.; Kodama, K.; Hasegawa, T. Modeling of shape memory alloy actuator and tracking control system with the model. IEEE Trans. Control Syst. Technol. 2001, 9, 54-59.

16. Dutta S.M.; Ghorbel, F.H. Differential hysteresis modeling of a shape memory alloy wire actuator. IEEE-ASME Trans. Mechatron. 2005, 10, 189-197.

17. Instruction Manual of Bio-Metal Fiber BMF150; Toki Corporation: Tokyo Japan, 2009.

18. Visintin, A. Differential Models of Hysteresis; Springer-Verlag: New York, NY, USA, 1995.

19. Dutta, S.M.; Ghorbel, F.H.; Dabney, J.B. Modeling and control of a shape memory alloy actuator. In Proceedings of the 20th IEEE International Symposium on Intelligent Control, Limassol, Cyprus, June 2005; pp. 1007-1012.

20. Elahinia, M.H.; Seigler, T.M.; Leo, D.J.; Ahmadian, M. Nonlinear stress-based control of a rotary SMA-actuated manipulator. J. Intel. Mat. Syst. Struct. 2004, 15, 495-508.

21. Holman, J.P. Heat Transfer, 8th ed.; McGraw-Hill Companies: New York, NY, USA, 1997.

22. Pons, J.L.; Reynaerts, D.; Peirs, J. ; Ceres, R.; VanBrussel, H. Comparison of different control approaches to drive SMA actuators. In Proceedings of 8th International Conference on Advanced Robotics, Monterey, CA, USA, July 1997; pp. 819-824.

(C) 2010 by the authors; licensee Molecular Diversity Preservation International, Basel, Switzerland. This article is an open-access article distributed under the terms and conditions of the Creative Commons Attribution license (http://creativecommons.org/licenses/by/3.0/). 